\title{
Retrospective Assessment of the Success Rate of Single-Visit Root Canal Treatment: A Clinical and Radiographical Analysis
}

\author{
Ali M. Rashid \\ BDS, MSc (Assist Lect)
}

\author{
Department of Conservative Dentistry \\ College of Dentistry, University of Mosul
}

\begin{abstract}
Aim: The purpose of this study was to determine retrospectively, clinically and radiographically the success rate of single-visit root canal treatment and determine the possible factors that could affected there prognosis.Materials and Methods: nine-hundred and sixty-five single-visi1 cases, of which 322 present for re-examination appointment ranging from 6 months to 5 years from the day of treatment were considered. Clinical and radiographical data were used to form overall impression of the outcomes for each case at the time of re-examination. Available demographics and treatment information of these 322 cases were compiled for comparison. The number of treatment visits was not determined by a pretreatment diagnosis or a re-assessment of the pulp status upon entry in to the tooth; therefore both vital and necrotic cases, as well as those with and without periradicular pathosis, Were included. Statistical analysis was carried out using Chi-square test and considered variations in failure rates based on gender, tooth type, position and arch. A $t$-test was used to evaluate data on age. Results: The overall success rate was $92.8 \%$. No statistically significan 1 differences were seen based on gender and arches. The data show almost younger ages more candidate for failure rate than older age group. Statistically, anterior teeth were more successful than posterior teeth. Conclusion: The success rate of single-visit root canal therapy was engorgement for this approach .Both gender and arches were not affecting the treatment outcome in this study, where as the treatment for older age and anterior teeth more successful than younger and posterior teeth respectively.

Key words: Retrospective study, Single-visit root canal therapy, Success.

Rashid AM. Retrospective Assessment of the Success Rate of Single-Visit Root Canal Treatment: A Clinical and Radiographical Analysis. Al-Rafidain Dent J. 2008; 8(1):104 -109

Received: 7/12/2006 Sent to Referees: 7/12/2006

Accepted for Publication: 11/6/2007
\end{abstract}

\section{INTRODUCTION}

In recent years one-appointment endodontic has gained increased acceptance as the best treatment for most cases. Some endodontists even feel there are few cases that cannot be treated successfully in one appointment ${ }^{(1,2)}$. Historically, root canal treatment was performed in multiple visits primarily to ensure 'sterility' of the root canal system prior to obturation ${ }^{(3)}$, and in between visits they advocated use of a wide variety of antimicrobial agents to eliminate microbes. In addition to killing bacteria, these agents, primarily phenolic compounds, were also highly irritating to the periradicular tissues ${ }^{(4)}$.

Those who believed that successful root canal treatment can be accomplished in one visit have rationale in the literature. Studies concerning postoperative pain ${ }^{(5,6)}$, as well as healing rates, shows the treatment outcomes to be similar, whether completed in one visit or in multiple visits ${ }^{(7)}$. Along with these advantages are the benefits of increased patient acceptance and limiting duplicate procedures ${ }^{(8)}$. Single-visit treatment means at least one fewer appointment, this decreases the number of operative procedures, including additional anesthesia, gingival trauma from rubber dam placement, as well as eliminating the risk of interappointment leakage through temporary restorations. These benefits, along with logistical patient management issues, such as loss of time from work and family, increase patient acceptance $^{(7,8)}$.

Those who advocated multiple-visit procedures proposed that the antimicrobial property of interappointment calcium hydroxide placement is required to ensure successful periradicular healing ${ }^{(7,9)}$, although predictable levels of bacterial reduction via refined cleaning and shaping techniques in one appointment may negate this need ${ }^{(10)}$. Furthermore, when flare-ups 
occur during multiple-visit procedures, they can be addressed prior to obturation (Soltanoff 1978) ${ }^{11}$. This is not an option in a single-visit treatment regimen. When flare-ups occur, non-surgical re-treatment or surgical intervention is usually necessary.

As a result, two divided schools of thought continue to exist concerning the number of visits necessary to achieve predictable success with root canal treatment. In more recent years studies have been published attempting to answer the basic question: Is single visit endodontic therapy more or less successful than endodontic therapy performed in multiple visits.

The purpose of this study was to determine retrospectively clinically and radiographically the success rate of single-visit root canal treatment and determine the possible factors that could affected the prognosis.

\section{MATERIALS AND METHODS}

Record of all patients seen in the Department of Conservative Dentistry, College of Dentistry, University of Mosul, Iraq and private endodontic clinic in the same city from 2001 to 2006 were secreened retrospectively for initiation and completion of non-surgical root canal treatment in one visit. This resulted in 965 single visit cases, of which $322(33.3 \%$ recall rate) presented for a re-examination appointment ranging from 6 month to 5 years from the day of treatment. Clinical and radiographical data gathered by practitioner were combined to form an overall decision of success or failure for each case at the time of re-examination. The number of treatment visits was not determined by any preoperative diagnosis; therefore, both vital and necrotic cases, as well as those with and without periradicular pathoses, were included. However, if the canals could not be dried, the tooth was not obturated in the first appointment and therefore was excluded from the data collection. Working lengths were obtained primarily by tactile sense and confirmation by radiograph. Instrumentation was completed in a crown-down manner with Ni-Ti Protaper hand instrument (Maillefer Instruments SA, Ballaigues, Switzerland ) or a combination of $\mathrm{Ni}-\mathrm{Ti}$ and stainless steel hand filing (Maillefer Instruments SA, Ballaigues, Switzerland ) according to Morgan and Montgomery $(1984)^{(12)}$. Irrigants used during the procedures included $5.25 \% \mathrm{NaOCl}$ and $2 \% \mathrm{H} 2 \mathrm{O} 2$. Obturation was completed with thermafil compactors (Maillefer Instruments SA, Ballaigues, Switzerland ) using sealar (Dorident, Austria ).

Only those patients who returned for re-examination appointments of 6 months or longer were included in the study unless further treatment of the tooth was initiated prior to this time, deeming these cases as failures. If the tooth was clinically and radiographically within normal limits (Gutman $1992^{(13)}$ ) table(1), the treatment was considered successful. If the tooth was symptomatic, provided no evidence of healing radiographically and required retreatment, surgical intervention or extraction, the case was considered a failure. Statistical analysis was carried out using Chi-square tests and considered variations in failure rates based on gender, tooth type, position and arch. For the data on age, a $t$-test was used to evaluate differences in failure rates. Significance was considered to be $p=0.05$.

\section{RESULT}

The study comprised of 322 patients (201 females and 121 males); Table (2). Of the females, 183 treatments $(91 \%)$ were successful and 18 treatments (9\%) failed. The males had $116(95.8 \%)$ successful and $5(4.2 \%)$ failed. No statistical differences were found based on gender, even though the failure rate was two times as high in females compared to that in males. ldeally, if a higher percentage of recall evaluations had been obtained, the data might have reflected different outcomes.

Patients in the study ranged in age from 15 to 61 years. Of the 299 successful cases in the study, the mean age was 31 years ( \pm 10 years) as compared to the mean age of 21 years ( \pm 9 years) in the failure group. The data show almost younger ages more candidates for failure rate than older age group. (table 3). Consequently, the $\mathrm{t}-$ test indicated there is a statistically significant difference in failure rates based up on age. 
Table (1) Guideline for clinical and radiographic success (adapted from Gutmann 1992).

\begin{tabular}{lll}
\hline Cuccess & Radinical & \\
& No tenderness to percussion or \\
palpation & $\begin{array}{l}\text { Normal to slightly thickened periodontal } \\
\text { ligament space }(<1 \mathrm{~mm})\end{array}$ \\
Normal mobility & Elimination of previous rarefaction \\
No sinus tract or periodontal dis- & $\begin{array}{l}\text { Normal lamina dura } \\
\text { ease }\end{array}$ & No evidence of resorption \\
Tooth function & $\begin{array}{l}\text { Dense, three-dimentional obturation of } \\
\text { canal space }\end{array}$ \\
No sign of infection or swelling & No evidence of subjective dis- & Extending to cementum-dentin junction \\
comfort & $(1 \mathrm{~mm}$ from apex $)$
\end{tabular}

Questionable

Sporadic vague symptomology, often not reproducible

Pressure sensation or feeling of fullness

Increased periodontal ligament space $(>1 \mathrm{~mm}$ and $<2 \mathrm{~mm})$

Stationary rarefaction or slight repair evident

Low-grade discomfort following percussion, palpation or chewing Discomfort when pressure is applied by the tongue

Superimposed sinusitis with focus on the treated tooth Occasional need for analgesic to relive minimal discomfort

Increased lamina dura space

Evidence of resorption

Void in obturation density

Extension of filling material beyond anatomic apex

failure

Persistent subjective symptoms

Increased width of periodontal ligament

Recurrent sinus tract or swelling space $(>2 \mathrm{~mm})$

Predictable discomfort to percussion or palpation

Lack of osseous repair within rarefaction or increased rarefaction

Evidence of irreparable tooth fracture

Lack of new lamina dura

Presence of osseous rarefacations in per-

Extensive mobility or progressive periodontal breakdown radicular areas where previously none existed

Inability to function on the tooth Visible, patent canal space-unfilled Excessive overextension with voids in apical third

Active resorption coupled other radiographic sigs of failure

Table (2) Success rate by gender.

\begin{tabular}{ccc}
\hline & $\mathrm{F}$ & $\mathrm{M}$ \\
\hline Success & $183(91 \%)$ & $116(95.8 \%)$ \\
Failure & $18(9 \%)$ & $5(4.2 \%)$ \\
\hline Total & 201 & 121 \\
\hline
\end{tabular}

F:femal;M:male 
Table (3) Success rate by age.

\begin{tabular}{lccc}
\hline & $\mathrm{N}$ & Mean & $\mathrm{SD}$ \\
\hline Success & 299 & 31.21 & 10.05 \\
Failure & 23 & 21.25 & 9.55 \\
\hline
\end{tabular}

Based upon tooth type and tooth position, teeth were divided between mandibular and maxillary arches resulting in 160 maxillary and 162 mandibular teeth included in the analysis (Table 4). The results show that treatment in 149
( 93\%) maxillary and 150 (92.3\%) mandibular teeth was successful. Therefore, this nearly even distribution showed equality in successful treatment of both maxillary and mandibular teeth.

Table (4) Success rate by archs.

\begin{tabular}{lllll}
\hline & Maxillary & Mandibular & Total \\
& & & \\
\hline Success & $149(93 \%)$ & 150 & $(92.3 \%)$ & 299 \\
Failure & $11(7 \%)$ & $12(7.7 \%)$ & 23 \\
\hline
\end{tabular}

With respect to type of tooth, 50 incisors, 102 premolars and 170 molars were evaluated; the success rates were 97, 91.5, and $89.3 \%$, respectively. The rates amongst these groups were not significant ( $p$ 0.075), however, the numerical trend showed that the incisors tended to have a much lower failure rate Table (5).

Table (5) Success rate by tooth type.

\begin{tabular}{llll}
\hline & Incisors & Premolar & Molar \\
\hline Success & $97 \%$ & $91.5 \%$ & $89.3 \%$ \\
Failure & $3 \%$ & $8.5 \%$ & $10.7 \%$ \\
\hline
\end{tabular}

In a similar comparison, the premolars and molars were combined into one 'posterior' grouping. When looking at the data this way, significant differences in failure rates were found between the groups. The anterior group had only 1 failure ( $2 \%$ ) compared to the failure of the 22 posterior teeth $(8 \%)$ Table (6). Regarding to the questionable cases, there are no one returned during

Table (6) Success rate by difference between anterior and posterior teeth.

\begin{tabular}{lll}
\hline & Anterior teeth & Posterior teeth \\
\hline Success & $49(98 \%)$ & $250(92 \%)$ \\
Failure & $1(2 \%)$ & $22(8 \%)$ \\
\hline
\end{tabular}

\section{DISCUSSION}

In assessing treatment outcomes by gender, females had a higher failure rate
(9\%) compared to males $(4.2 \%)$. These differences, however, were not statistically significant. Because there were 201 females 
and only 121 males in the study, it would be appear that females were more conscientious about returning for their follow-up examinations than males; however, this may not be accurate. Of the 965 cases of single-visit root canal treatment, 201 were females and 121 were males. This is about a 2: 1 ratio of those that were initially treated, which is similar to the re-examination rate. These findings are similar to those by Smith ${ }^{(14)}$, who also found more females to present for root canal treatment, but a lower percentage of success in women than in men. However, Soikkonen ${ }^{(15)}$ took radiographs of patients and found more periradicular radiolucencies present in men than in women. These findings, however, were seen in teeth with root canal treatment, as well as in those patients who had never been treated endodontically.

The age of the patient was evaluated because of the inherent good prognosis for the older patient is actually better than that for the younger age group on a statistical basis. This is probably because of the tighter apical foramina, lack of completely patent auxiliary canals, dense preiapical bone ${ }^{(16)}$. Although on the other hand the difficulties encountered in teeth in which canals, through time, continue to narrow down as a result of deposition of mineralized tissue ${ }^{(17)}$, as well as the decrease in healing ability of elderly patients ${ }^{(18)}$. Despite these physiological differences, the age of the patient was appear to affect the outcome of treatment.

Accumulation of chronic illnesses is the major factor in healing delays of the elderly ${ }^{(19)}$. The patients' systemic health status was not able to be collected retrospectively; however, all patients were treated in a private practice setting, which would include mostly healthy patients and those with minor health concerns. This could explain the similarities in healing as related to age.

Treatment considerations change depending on the complexity of each tooth. Some of these teeth include anatomical variations as seen radiographically, clinically and those understood from studying similarities in tooth type, as well as number of canals and/or roots ${ }^{(17)}$. Therefore, the data were evaluated in several different ways to compare the success rates by position and type of tooth.

The first analysis differentiated success rates between maxillary and mandibular teeth. The groups were divided evenly having 162 mandibular and 160 maxillary teeth evaluated,these results correspond to those found by Pekruhn ${ }^{(19)}$ in that the failure rate of all maxillary teeth was $5.4 \%$ compared to $5.0 \%$ in mandibular teeth, even though the ratio of maxillary versus mandibular teeth was around 2: 1 (607 maxillary, 318 mandibular).

Another comparison was by tooth type. Each tooth type, whether they were mandibular or maxillary, was classified into three groups: incisors, premolars and molars (table 6). As only two incisors of 50 included in the study failed, the success rate was numerically higher $(97 \%)$ than the premolars and molars (91.5 and 89.3\%, respectively). The $P$-value was closed to achieving statistical significance $(P=0.078)$, which may or may not have been attined if the sample size available had larger. The result resemble the result obtained by Rudner \&Oliet ${ }^{(20)}$ who found slight increases in success when going from molars to anterior teeth $(85.7 \%$ in molars, $90.4 \%$ in premolars and $91.8 \%$ in anterior teeth).

When comparing anterior teeth to premolars and molars in one 'posterior teeth' group (table 5), the Chi-square analysis showed statistical significance $(P=0.0124)$. the difference is most likely seen because of anatomical complexities of posterior teeth compared to the single-rooted canal systems of anterior teeth. However, Rudner \&Oliet 1981 found that no difference between anterior and posterior group.

\section{CONCLUSION}

The success rate of single-visit root canal therapy was engorgement for this approach, according to this study. Both gender and arches were not affecting the treatment outcome, where as the treatment for older age and anterior teeth more successful than younger and posterior teeth respectively.

\section{REFERENCES}

1. Holland R, Filho JAO, de Souza V, Nery MJ A comparison of one versus two appointment endodontic therapy. $J$ Endodon 2003; 29: 121-129.

2. Inamoto K, Kojima K, Nagamatusu K, Hamaguchi A Asurvay of the incidence of single-visit endodontics. $J$ Endodon 2002; 28: 371-377. 
3. Bender IB, Senzer SI To culture or not to culture' Oral Surg, Oral Med and Orol Paho. 1964; 18, 527-40.

4. Koontongkaew S, Silapichit R,Thaweboon BI Clinical and laboratory assessments of camphorated Monochlorophenol in endodontic therapy. Oral Surg, Oral Med and Oral Patho 1988;65, 757-62.

5. Fava LR A clinical evaluation of one and two-appointment root canal therapy using calcium hydroxide. Inter. Endodon $J$ 1994 27, 47-51.

6. Eleazer PD, Eleazer KR: Flare-up rate in pulp after necrotic molars in one-visit versus two-visit endodontic treatment. $J$ Endodon 1998; 524, 614-6.3

7. Weiger R, Rosendahl R, Lost C Influence of calcium hydroxide intraoanal dressings on the prognosis of teeth with endodontioally induced periapical lesions. Inter. Endodon J. 2000; 33, 219-26.

8. Katebzadeh N, Sigurdsson A, Trope M Radiographic evaluation of periapical healing after obturation of infected root canals: an in vitro study. Inter. Endodon J $2000 ; 133,60-6$.

9. Trope M, Delano EO, Orstavik DI Endodontic treatment of teeth with apical periodontitis single vs. multi visit treatment. $J$ Endodon 1999; 525, 345-50

10. Card SJ, Sigurdsson A, Orstavik D, Trope MI The effectiveness of increased apical enlargement in reducing intracanal bacteria. J Endodon 2002; 779-83 11.
11. Morgan LF and Montgomery S An evaluation of the crown-down pressureless technique. J. Endodon 1984. 10: 491498.

12. Gutman JL Clinical, radiographic, and histologic prespective on success and failure in endodontics. Dent Clinics of North America; 1992 36, 379-92.

13. Smith CS, Setchell DJ, Harty FJ Factors influencing the success of conventional root canal therapy-a 5-year retrospective study. Inter. Endodo J 1993; 126, 321-83.

14. Soikkonen K Endodontioally treated teeth and preiapical findings in the elderly. Inter. Endodontic J 1995; 28, 200-8.

15. Dugas NN, Lawrence HP, Teplitsky P, Friedman S Quality of life and satisfacation outcomes of endodontic treatment. J Endodon; 2002; 28: 819-24.

16. Dummer PM, McGinn JH The position and topography of the apical canal constriction and apical foramen. Inter. Endodo J 1984 17, 192-8.

17. Williams M, Hadler NM Sounding board The illness as the focus of geriatric medicine. New England J of Medicine 1983; 308, 1357-60.

18. Pekruhn RB The incidence of failure following single-visit endodontic therapy. J Endodon 1986; 512, 68-72.

19. Rudner WL, OIiet S Single-visit endodontics: a concept and a clinical study. Compendium of Continuing Educ Mion in Denti5try 1986; 2, 63-8. 